\title{
HIGH VELOCITY CLOUDS IN THE GALAXY
}

\author{
FELIX J. LOCKMAN \\ National Radio Astronomy Observatory ${ }^{\dagger}$ \\ P.O. Box 2 \\ Green Bank, WV 24944 USA \\ (jlockman@sadira.gb.nrao.edu)
}

\section{Introduction}

Early observers measuring $21 \mathrm{~cm}$ HI profiles away from the Galactic plane found not only the emission near zero velocity expected from gas in the immediate vicinity of the Sun, but also occasional emission at velocities reaching several hundred $\mathrm{km} \mathrm{s}^{-1}$. It seemed unlikely that these spectral lines could come from gas in normal galactic rotation (they are sometimes found at $|b|>45^{\circ}$ ), and so began the puzzle of "high-velocity clouds" (HVCs). The early result that all HVCs had negative velocity implying that they were infalling was soon shown to be incorrect with the discovery of many positive velocity clouds in the southern hemisphere. Attempts to determine the distance to HVCs by searching for them in absorption against stars yielded only lower limits, typically $>1 \mathrm{kpc}$. By 1984 several large-scale surveys had established that a significant fraction of the sky was covered with high velocity HI (e.g., Oort, 1966; Giovanelli, 1980). A recent major review is by Wakker (1991a; see also van Woerden, 1993). For this brief presentation to a specialized audience, I will concentrate on issues that may be relevant to the topic of stellar populations.

\section{A few Comments about the Disk}

In recent years we have learned that there are considerable amounts of gas following Galactic rotation many scale-heights above the plane (reviewed in Dickey and Lockman, 1990). Sometimes the high-z gas is recognizable

\footnotetext{
${ }^{\dagger}$ The National Radio Astronomy Observatory is operated by Associated Universities, Inc., under a cooperative agreement with the National Science Foundation
} 
as part of a "supershell" probably launched to its height by numerous overlapping supernova remnants (Heiles, 1979, but also see Mirabel, 1991). Segments of shells with $>10^{4} M_{\odot}$ of HI are found half a kpc or more from the Galactic plane. Some shells have additional mass in molecular gas, and some may even contain regions dense enough to initiate star formation. Few HI observers would be shocked to find the occasional O or B star, or other evidence of recent star formation, in the lower galactic halo.

In a related development, the large-scale warping and flaring in the gaseous layer, which begins just a bit outward from the Sun, has been found to be more extensive than previously thought. At some longitudes the warped disk can be traced to $|b|>30^{\circ}$ (Diplas and Savage, 1991) implying a distance from the plane of several kpc. It seems certain that some HI features at one time classified as HVCs are part of the Galactic disk, as suggested, e.g., by Davies (1972) and Verschuur (1975).

\section{HVC Metallicity}

High-velocity clouds have been difficult to detect in optical absorption lines against distant stars, and for a time, this was thought to indicate that they might be metal-poor rather than distant. In recent years, though, many HVCs have been detected in optical and UV absorption lines against extragalactic objects (e.g. de Boer et al., 1991). Table 1 gives an incomplete summary of recent data on HVC abundances. Although the uncertainties are still large, it is clear that HVCs are not composed of primordial gas. Gas known to be in the galactic halo is also metal-rich (e.g., Albert et al., 1993), and the scale-height of species like CaII and TiII is greater than that of hydrogen, no doubt because of decreased depletion away from the plane. However, HVCs are still somewhat unique in that they have much less farIR emission than comparable amounts of low- and intermediate-velocity HI. This implies that they may be deficient in dust compared to disk HI or that their dust is cooler (Wakker and Boulanger, 1986).

TABLE 1. Recent Abundance Determinations in HVCs Species $\log [$ abundance/H] Reference

\begin{tabular}{lcl}
\hline Si & $-1 \rightarrow-1.4$ & Danly et al., 1993 \\
MgII & $-0.5 \rightarrow-1.2$ & Savage et al., 1993 \\
MgII & $-2.0 \pm 0.1$ & Bowen and Blades, 1993 \\
MgII & $+0.4 \pm 0.4$ & Bowen and Blades, 1993 \\
$\mathrm{S}$ & -0.8 & Lu et al., 1994 \\
\hline
\end{tabular}




\section{Morphology and Sky-Covering Fraction}

High-velocity HI is not scattered randomly across the sky but is usually found in large, $>100^{\circ}$, complexes that have a smoothly varying velocity field. Wakker (1991b) concluded that about $18 \%$ of the sky is covered with HVC gas, and that most of it is part of one complex or another.

But when Savage et al. (1993) analyzed the Milky Way ultraviolet absorption lines that were detected in the initial phase of the HST survey of quasar absorption lines, their rather startling result was that about half the site-lines (7 of 15) showed high-velocity Galactic MgII in absorption. This high fraction could be a vagary of small number statistics, or it could imply that there is a population of HVCs detectable in MgII but not in HI at the sensitivity level of existing $21 \mathrm{~cm}$ surveys. Prompted by this, Murphy, Lockman and Savage (1995) searched for $21 \mathrm{~cm}$ HVCs in several hundred high-latitude directions to a completeness limit of $7 \times 10^{18} \mathrm{~cm}^{-2}$, a factor $\sim 5$ lower limit than previous work. Preliminary results support two conclusions: 1) at the greater sensitivity level about $37 \%$ of the high-latitude sky is covered with high-velocity HI, double the fraction known before, and 2) virtually all of the newly-discovered, low- $\mathrm{N}_{H}, \mathrm{HVCs}$ can be associated with one of the major HVC complexes.

The second conclusion is perhaps the most important, for it implies that the study of HVCs is the study of the large complexes, and that the HI data do not show evidence for a haze of small clouds falling into the Milky Way. It also implies that the newly discovered gas has about the same distance as the bright complexes and thus its mass can be estimated relative to that of the brighter emission. It turns out to be negligible. There is an excellent chance that we already have observed virtually all of the HI mass involved in the HVC phenomenon.

\section{What are They?}

Because the distance to most HVCs is unknown (see Danly et al., 1993, for a rare exception) various scenarios have been proposed to explain them. These models (reviewed in Wakker, 1991a) include: return of gas that has been expelled into the halo by a Galactic fountain; material stripped from the Magellanic clouds by their encounter with the Milky Way; infalling gas that is being accreted by the Galaxy and the remnants of this interaction; gas that is part of warped distant spiral arm; and a nearby very-large supernova explosion. If there is a consensus in this field it is that HVCs are a heterogeneous population, but one which is primarily associated with our Galaxy, and not with intergalactic or pregalactic conditions. Moreover, since the dominant HVC structures are large and metal-rich, they may be just misunderstood or misclassified parts of the Galaxy's gaseous disk. With 
the recent implication that perhaps most of the sky contains high-velocity material, the size of our Galaxy may in fact be defined by the HVCs, and not by disk gas.

\section{References}

Albert, C.E., Blades, J.C., Morton, D.C., Lockman, F.J., Proulx, M. and Ferrarese, L., 1993, Ap. J. Suppl. 88, 81

Bowen, D.V. and Blades, J.C., 1993, Ap. J. 403, L55

Danly, L., Albert, C.E. and Kuntz, K.D. 1993, Ap. J. 416, L29

Davies, R.D., 1972, Nature, 237, 88

de Boer, K.S., Herbstmeier, U. and Mebold, U., 1991, The Interstellar Disk-Halo Connection in Galaxies, IAU Symposium 144, ed. H. Bloemen, Kluwer, p.161

Dickey, J.M. and Lockman, F.J., 1990, Ann. Rev. Astron. Astroph. 28, 215

Dieter, N. H., 1964, A. J. 69, 288

Diplas, A. and Savage, B.D., 1991, Ap. J. 377, 126

Giovanelli, R., 1980, A. J. 85, 1155

Heiles, C.E., 1979, Ap. J. 229, 533

Lu, L., Savage, B.D. and Sembach, K.R., 1994, Ap. J. 426, 563

Mirabel, I.F., 1991, The Interstellar Disk-Halo Connection in Galaxies, IAU Symposium 144, ed. H. Bloemen, Kluwer, p.89

Murphy, E.M., Lockman, F.J. and Savage, B.D., 1995, Ap. J., in press

Oort, J.H., 1966, Bull. Astron. Neth. 18, 421

Savage, B.D. et al., 1993, Ap. J. 413, 116

Schulman, E., Bregman, J.N. and Roberts, M.S., 1993, Ap. J. 423, 180

van der Hulst, J.M. and Kamphuis, J., 1991, The Interstellar Disk-Halo Connection in Galaxies, IAU Symposium 144, ed. H. Bloemen, Kluwer, p.201

van Woerden, H., 1993, Luminous High-Latitude Stars, ed. D.D. Sasselov, AIP Conference Series vol. 45, p 11

Verschuur, G.L., 1975, Ann. Rev. Astron. Astroph. 13, 257

Wakker, B.P., 1991a, The Interstellar Disk-Halo Connection in Galaxies, IAU Symposium 144 , ed. H. Bloemen, Kluwer, p.27

Wakker, B.P., 1991b, A. \& A. 250, 499

Wakker, B.P. and Boulanger, F., 1986, A. \& A. 170, 84

PECKER: You emphasized the fact that HVCs were discovered in Holland, and widely publicized, notably by Oort, who deserves a large part of the credit. But at about the same time, Nannielou Dieter, at Berkeley, had seen the infalling hydrogen; she called it "the belt" and she insisted it covered a large part of the sky, perhaps about $1 / 3$, thus agreeing with what you have shown to us today.

LOCKMAN: Yes, Dieter's work was important. She was one of the first to suggest that the negative velocity gas near the North Galactic Pole might be evidence of clouds condensing from hot gas in the halo (Dieter, 1964).

R. WYSE: Could you comment on related phenomena in external galaxies?

LOCKMAN: Most high-resolution HI observations of other galaxies lack the sensitivity to detect any but the brightest HVCs, but some are found (see van der Hulst and Kamphuis, 1991). Also, a large fraction of face-on galaxies have highvelocity wings on their HI profiles (Schulman et al., 1993). The comparison should be clearer in the next few years. 